\title{
ELECTRICAL AND OPTICAL PROPERTIES OF AMORPHOUS TIN OXIDE
}

I. Chambouleyron, C. Constantino*, D. Jousse**, R. Assumpção and

R. Brenzikofer.

Physics Institute, UNICAMP, C.P. 1170, Campinas, S.P., 13.100 Brazil

\begin{abstract}
Thin layers of tin oxide have been deposited by the chemical spray method. From $x$-ray diffraction, Hall effect and optical measurements we conclude that films deposited at temperatures lower than $300^{\circ} \mathrm{C}$ are amorphous. Transport and optical data are given for both polycrystalline and amorphous materials.
\end{abstract}

INTRODUCTION.- Electrical and optical properties and structure of single crystal tin dioxide have been investigated both theoretically and experimentally. Films grown under different conditions and with different methods have been extensively studied too. To the authors knowledge no such properties have been reported yet on amorphous tin oxide. We present here preliminary results on the influence of deposition temperature on the X-ray diffraction patterns, Hall mobility and carrier concentration and the optical absorption in the fundamental edge region of the spectrum. The properties of the films are strongly dependent on annealing processes in air or vacuum. Changes of several orders of magnitude in resistivity have been reported to occur in annealed samples. In this paper we report measurements on "as grown" samples only. The time elapsed while deposition is made also influences the layer properties.

FILM GROWTH AND STRUCTURAL PROPERTIES. - SnO layers were grown on glass and quartz flat substrates. All films were obtained by the chemical spray method using ethanol as a solvent in the sprayed solution. The substrate temperature $T_{s}$ at which we deposited our samples was allowed to vary between 260 and $440^{\circ} \mathrm{C}$. S Between those limits films of relatively good transparency were obtained, although with important differences in their structural, electrical and optical properties. Typical deposition parameters are as follows: carrier gas (nitrogen) flow: $7 \mathrm{l} / \mathrm{min}$; deposition rate: $1 \mathrm{ml} / \mathrm{min}$. The sprayed solution volume is always $25 \mathrm{ml}$. St:bstrate temperature was continuously monitored with a chromel alumel thermocouple. The experimental set-up is essentially the same as given in (1).

Tin dioxide crystallizes in the tetragonal structure with coordination number $6: 3$. The ideal reaction for the pyrolitic decomposition of stannic chloride is

$$
\mathrm{SnC} \ell_{4}+2 \mathrm{H}_{2} \mathrm{O} \rightarrow \mathrm{SnO}_{2}+4 \mathrm{HCl}
$$

This is likely to happen at temperatures higher than $500^{\circ} \mathrm{C}$ and the composition of the films is not very far from stoichiometry. When the temperature is lowered the reaction is incomplete. Full oxidation of tin does not occur and atomic chlorine and hydrogen are included into the lattice. Secondary Ion Mass Spectroscopy and Auger electron spectroscopy of layers grown at $260^{\circ} \mathrm{C}$ show a large concentration of chlorine that amounts to a few atomic percent (Chambouleyron and Farias, unpublished).

Optical microscopy shows that low temperature deposited films are somewhat

* Supported by FAPESP; ** On leave from CNRS, France. 
inhomogeneous. As the substrate temperature increases homogeneity improves. It is possible that when deposition temperature is low, small adatom surface mobility and a large concentration of foreign atoms produce a highly inhomogeneous material.

X-ray diffraction patterns were obtained with a $12 \mathrm{KW}$ Rigaku Rotaflex rotating anode diffractometer. They give indications of the degree of crystallinity and were used among other criteria, to detect the amorphous nature of the films. It appears that deposition temperatures lower than around 3000 produce films with a very poor degreee or the absence of crystallinity. On the grounds of their optical and electrical properties we believe these films are amorphous.

TRANSPORT PROPERTIES.- Electrical conductivity and Hall effect measurements were performed on 0.5-1 $\mathrm{\mu m}$ thick layers using the Van der Pauw geometry. Some samples have also been characterized by varying the measurement temperature from liquid nitrogen to room temperature.

The conductivity reaches a remarkable plateau value of $130(\Omega \mathrm{cm})^{-1}$ at $300 \%$ in the $\mathrm{T}$ range $\left\{300^{\circ} \mathrm{C}, 400^{\circ} \mathrm{C}\right\}$. Conductivity decreases drastically of two orders of magnitude for $T_{s}$ lower than $300^{\circ} \mathrm{C}$.

The sign of the Hall potential is the same for all samples and indicate a n-type conductivity. Figure 1 shows the density of free carriers $n$ as deduced from the usual Hall constant formula $\mathrm{R}_{\mathrm{H}}=-1 /$ ne that we assume to apply in all materials. The experimental difficulty in determining the small Hall potentials (10-50 $1 \mathrm{~V})$ lead to important uncertainties for $n$ especially in the case of higher resistivity samples. All macerials are either highly degenerated $\left(300^{\circ} \mathrm{C} \leqslant \mathrm{T} \leqslant 440^{\circ} \mathrm{C}\right)$ or 1 ightly degenerated $\left(T \leqslant 280^{\circ} \mathrm{C}\right)$. Assuming a value of $0.35 \mathrm{~m}_{0}$ (2) for the $\mathrm{s}$ electronic density of states effective mass and a parabolic band model, the effective density of states

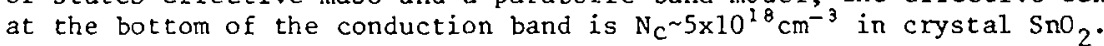

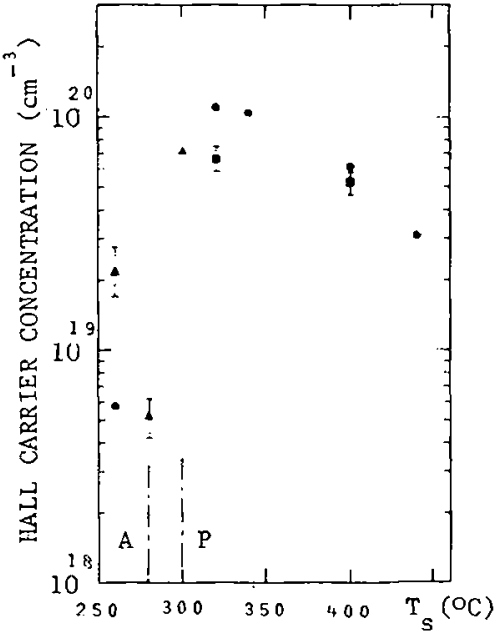

Fig. I - Hall carrier concentrations versus substrate temperature of amorphous (A) and polycrystalline (P) tin oxide for various solution fluxes ( intermittent $1.5 \mathrm{~m} \ell / \mathrm{min}$, - cont. $4 \mathrm{~m} \ell / \mathrm{min}, \mathbf{c o n t} .1 \mathrm{~m} \ell / \mathrm{min}$.$) .$

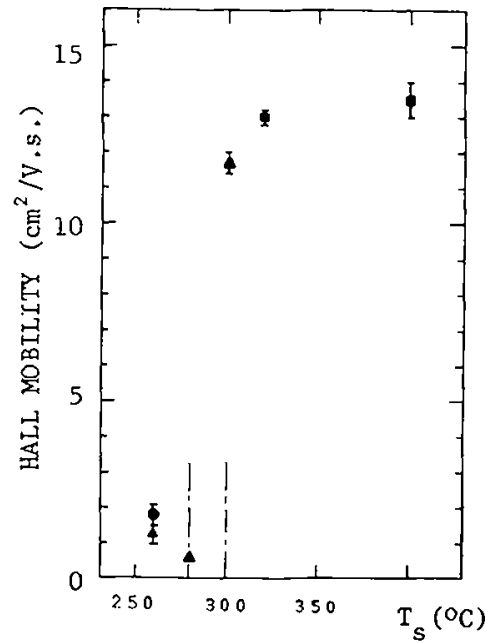

Fig. 2 - Electron Hall mobility versus substrate tempe rature of some samples of $\mathrm{fig} \cdot \overline{\mathrm{I}}$.

Hall mobilities $\mu_{H}$ are represented in fig.2. As the deposition temperature goes down below $300^{\circ} \mathrm{C}$, the mobility drops an order of magnitude from the usual polycrystalline value of $10-15 \mathrm{~cm}^{2} / \mathrm{V}$.s. This drop gives some evidence of a transition towards an amorphous state. Measured HH values 
are consistent with conduction through extended states. Following (3) the usual Hall treatment appears to remain valid for a degenerate amorphous material. According to Friedman (4) the value of $\mu_{\mathrm{H}}$ is expected to be smaller than the conductivity mobility in extended states $j_{c}$. Let us remark that an underestimation of $\mu_{c}$ from Hall measurements lead to an overestimation of $n$. This would imply that the $n$ value measured for our amorphous samples shown in figure 1 do not correspond to true carrier concentrations. Actual values may be near to or lower than the $10^{18} \mathrm{~cm}^{-3}$ degeneracy value. Indeed, temperature dependence of conductivity performed on two materials prepared at $320^{\circ} \mathrm{C}$ and $260^{\circ} \mathrm{C}$ enlightens this point. As shown in figure 3 conductivity in polycrystalline tin oxide $\left(\mathrm{T}_{S}=320^{\circ} \mathrm{C}\right)$ does not vary with temperature as expected for a semiconductor in which the Fermi level 1 ies far inside the conduction band. On the contrary, the amorphous tin oxide conductivity increases with temperature which suggests at first glance that the Fermi level lies below the conduction mobility edge $E_{C}$. In our preliminary experiments no activation energy

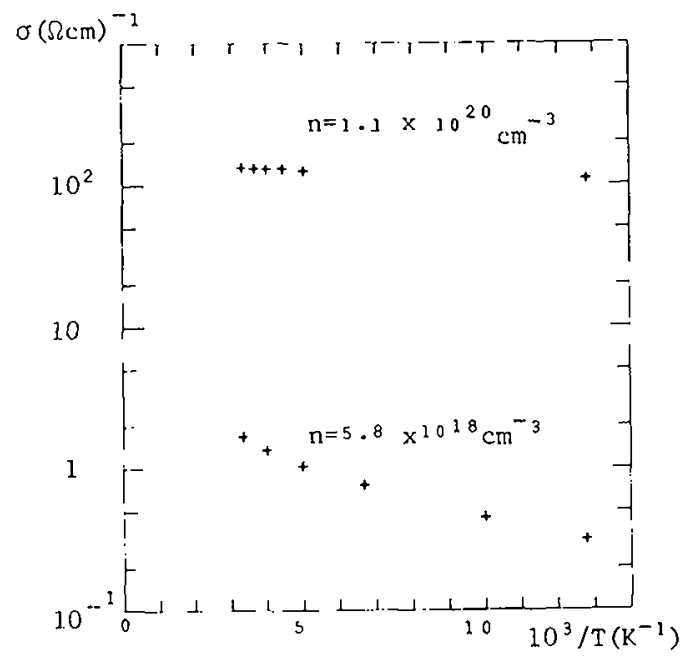

Fig. 3 - DC Conductivity of amorphous $\left(T_{s}=260^{\circ} \mathrm{C}\right)$ and polycristalline $\left(\mathrm{I}_{\mathrm{s}}=320^{\circ} \mathrm{C}\right)$ materials versus $1 / \mathrm{T}$. has been clearly detected at high temperatures. However a linear dependence of $\log \sigma$ vs $\mathrm{T}^{-1 / 4}$ is obtained at temperatures lower than $250^{\circ} \mathrm{K}$. This would indicate a hopping conduction process through localized states around the Fermi level (3).

A Fermi-level situated very near of $E_{c}$ accounts for the great sensibility of the electron densities to preparation conditions when $\mathrm{T}_{\mathrm{S}}<300^{\circ} \mathrm{C}$ (figure 1). Indeed, the creation of defect or impurity states in the gap occuring at low $\mathrm{T}_{\mathrm{S}}$, as evidenced from optical measurements is most probably responsible for the downwards shift of the Fermi-level.

OPTICAL ABSORPTION.- Optical transmission and reflection were measured with Zeiss DMC-25 and Beckmann DK 2 A spectrophotometers in the U., V., visible and near I.R. range of the spectrum. From interference patterns we could estimate the thickness of the films. Mechanical Talystep measurements confirmed the

optically deduced values. Absorption coefficents were calculated from transmission and reflection measurements. Independent values were obtained from films identical excent for thickness using the relation

$$
\alpha=\frac{1}{\Delta t} \ln \frac{\mathrm{I}_{1}}{\mathrm{I}_{2}}
$$

where $\Delta t=t_{2}-t_{1}$ is the film thickness difference and $I_{1}$ is the transmission through the thinner layer.

Figure 4 shows a logarithmic plot of absorption coefficient vs photon energy for samples grown at $320^{\circ}$ and $280^{\circ} \mathrm{C}$, compared to single crystal material $(5,6)$. The absorption coefficientof sprayed tin oxide layers shows some important differences with respect to crystalline material. On the one hand $\alpha$ is higher for amorphous material in the whole range. On the other hand both materials show an absorption coefficient near the fundamental edge that has an exponential type dependence on photon energy. As the deposition temperature increases and the material becomes more ordered the absorption edge evolves towards a curve having two different regions, an exponential shape on 
the low photon energy side and a parabolic type dependence at high photon energies.

An absorption coefficient that increases exponentially with photon energy has been measured on a great number of crystalline and in almost all amorphous semiconductors.

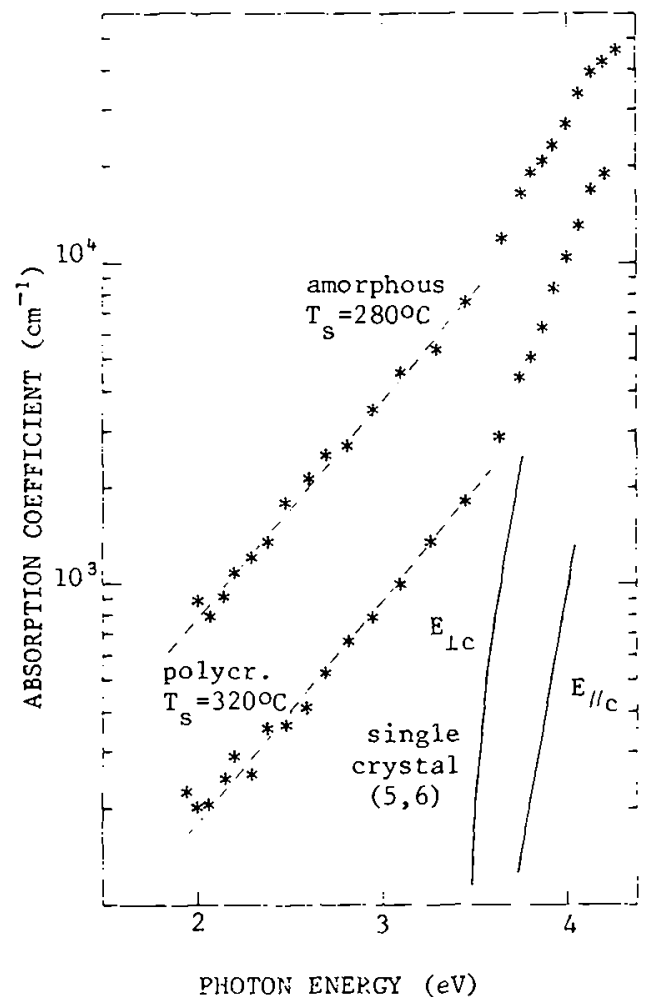

No single explanation has been accepted for this behaviour. Our preliminary results indicate however that two contributions might be responsible for the observed shape. One is an electric field broadening of the absorption edge (Franz-Keldysh effetc). The internal field could arise in principle from charged impurity states. The exponential behaviour might also be due to electronic transitions between tails of states in the band edge that fall off exponentially with energy. This interpretation is consistent with the experimentally observed fact that higher deposition temperatures give lower absorption in the tails. With increasing deposition temperature two mechanisms should occur: impurity atom concentration ( $\mathrm{C} \ell$ and $\mathrm{H}$ ) and deviations from stoichiometry (incomplete oxidation of tin) decrease and crystalline order is improved. Both mechanisms "clean" the forbidden band.

Fig.4 - Absorption coefficient versus photon energy for amorphous, polycrystalline and single crystal tin oxide.

ACKNOWLEDGEMENTS.- We thank I. Torriani and M. Fantini for x-ray diffraction measurements. This work has been partially supported by Companhia Energética de São Paulo.

\section{REFERENCES}

1. I. CHAMBOULEYRON and E. SAUCEDO, Solar Energy Materials, 1, (1979), 299.

2. NAGASAWA, SHIONOYA and MAKISHIMA, J. Phys. Soc., Japan, $2 \overline{0}(1965), 1093$.

3. N. F. MOTT and E. A. DAVIS, Electronic Process in Non-Crystalline Materials, Clarendon Press, Oxford, (1979).

4. L. FRIEDMAN, J. Non-Crystalline Solids, 6, (1971), 329.

5. S. REDDAWAY and D. WRIGHT, Brit. J. Appl. Phys. 16 (1965), 195.

6. SUMMIT, MARLEY and BORELli, J. Phys.\& Chem. Solids, 25 (1964), 1465. 